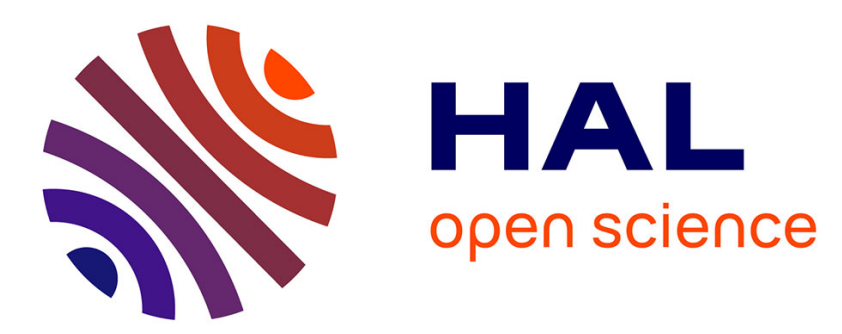

\title{
Modélisation du SQUID rf en réflectométrie: impédance à la fréquence de pompe, influence du bruit de fond
}

Didier Robbes, C. Dubuc, J. Lepaisant, Daniel Bloyet

\section{To cite this version:}

Didier Robbes, C. Dubuc, J. Lepaisant, Daniel Bloyet. Modélisation du SQUID rf en réflectométrie: impédance à la fréquence de pompe, influence du bruit de fond. Revue de Physique Appliquée, 1987, 22 (11), pp.1389-1398. 10.1051/rphysap:0198700220110138900 . jpa-00245689

\section{HAL Id: jpa-00245689 https://hal.science/jpa-00245689}

Submitted on 1 Jan 1987

HAL is a multi-disciplinary open access archive for the deposit and dissemination of scientific research documents, whether they are published or not. The documents may come from teaching and research institutions in France or abroad, or from public or private research centers.
L'archive ouverte pluridisciplinaire HAL, est destinée au dépôt et à la diffusion de documents scientifiques de niveau recherche, publiés ou non, émanant des établissements d'enseignement et de recherche français ou étrangers, des laboratoires publics ou privés. 


\title{
Modélisation du SQUID rf en réflectométrie : impédance à la fréquence de pompe, influence du bruit de fond
}

\author{
D. Robbes, C. Dubuc, J. Lepaisant et D. Bloyet \\ G.E.R.S.I.C., Institut des Sciences de la Matière et du Rayonnement, boulevard du Maréchal-Juin, 14032 \\ Caen Cedex, France
}

(Reçu le 16 juin 1987, accepté le 29 juillet 1987)

\begin{abstract}
Résumé. - Les échanges d'énergie d'un circuit bouchon et d'un SQUID rf sont considérés dans cet article en termes d'impédance équivalente placée en série avec la bobine d'injection. L'analyse harmonique à la fréquence de pompe $f_{\mathrm{P}}$ de la sonde classique attaquée en courant est d'abord précisée ; cette méthode, valide pour un SQUID largement hystérétique et faiblement couplé $(k \ll 1)$ à un circuit oscillant de coefficient de qualité $Q \gg 1$, donne une réponse en tension qui varie de façon discontinue et parfois multivaluée en fonction du niveau radiofréquence. La sonde classique est ensuite analysée directement dans le domaine temporel pour des SQUIDs suffisamment hystérétiques sans restrictions sur les valeurs des paramètres $k, Q, f_{\mathrm{P}}$. Les résultats précédents sont appliqués au cas de la réflectométrie $\left(f_{\mathrm{P}}=80 \mathrm{MHz}\right)$ en régime idéal puis bruyant ; nous montrons que le bruit intrinsèque du micropont est responsable de la déformation des caractéristiques idéales tandis que les fluctuations intrinsèques déduites de cette déformation conduisent à une densité spectrale de $1,1 \times 10^{-5} \phi_{0} / \sqrt{\mathrm{Hz}}$ et sont toujours dominées par les fluctuations induites par les sources externes. La sensibilité de notre système est de $10^{-4} \phi_{0} / \sqrt{\mathrm{Hz}}$. Les études d'optimisation montrent que la sonde adaptée autorise une grande souplesse dans le choix de $k$ et $Q$ sans amélioration ni détérioration notables du rapport signal sur bruit.
\end{abstract}

\begin{abstract}
Energy exchanges between a rf SQUID with a Dayem microbridge and the tank circuit coil are viewed in terms of an equivalent impedance placed in series with the coil. This allows to characterize the matched configurations which notably differ from the classical probe. In our case the tank circuit is pumped at $80 \mathrm{MHz}$ by an incident voltage wave and the reflected wave is synchronously detected. The harmonic analysis at the pumping frequency $f_{\mathrm{P}}$ of the classical probe is stated precisely; the method is valid for a large hysteretic mode of the rf SQUID, a weak coupling factor $k$ and a large quality factor $Q$. The deduced staircase pattern $V_{\mathrm{rf}}-I_{\mathrm{rf}}$ is found discontinuous and eventually multiple-valued. A time analysis of the classical probe is also presented which gives consistent results without any restrictions on the parameters $k, Q, f_{\mathrm{P}}$ values. The previous methods are then used to analyse the ideal and noisy running of the matched probe ; ideal curves are mainly distorted by the intrinsic noise of the microbridge. The deduced intrinsic fluctuations correspond to a spectral density of $1.1 \times 10^{-5} \phi_{0} / \sqrt{\mathrm{Hz}}$ while external noises sources give rise to the final flux sensibility of $10^{-4} \phi_{0} / \sqrt{\mathrm{Hz}}$. Optimization work states that for the matched probe, a large choice of $k, Q$ parameters is allowable without improvement nor decrease of the signal to noise ratio.
\end{abstract}

\section{Introduction.}

La configuration classique de mise en œuvre des SQUIDs rf (excitation en courant du circuit oscillant couplé au SQUID) devient inadéquate dès que la fréquence de pompe dépasse quelques dizaines de mégaHertz. Elle peut être avantageusement remplacée par une technique de réflectométrie $[1,2]$. La sonde classique est alors « adaptée » à une ligne de transmission au moyen d'une bobine d'inductance
$L_{\mathrm{A}}$ de résistance série $R_{\mathrm{A}}$ (Fig. 1). Ce montage d'une grande simplicité offre les avantages suivants :

- Le SQUID est excité en radiofréquence par une onde de tension incidente $V_{\mathrm{i}}$ qui est indépendante du coefficient de réflexion sur la sonde si les accès du coupleur directif sont adaptés.

- L'onde de tension $V_{\mathrm{r}}$ réfléchie par la sonde, séparée de l'onde incidente par un coupleur directif, contient sous forme de modulations d'amplitude et de phase, l'information sur l'état de flux quasistati- 


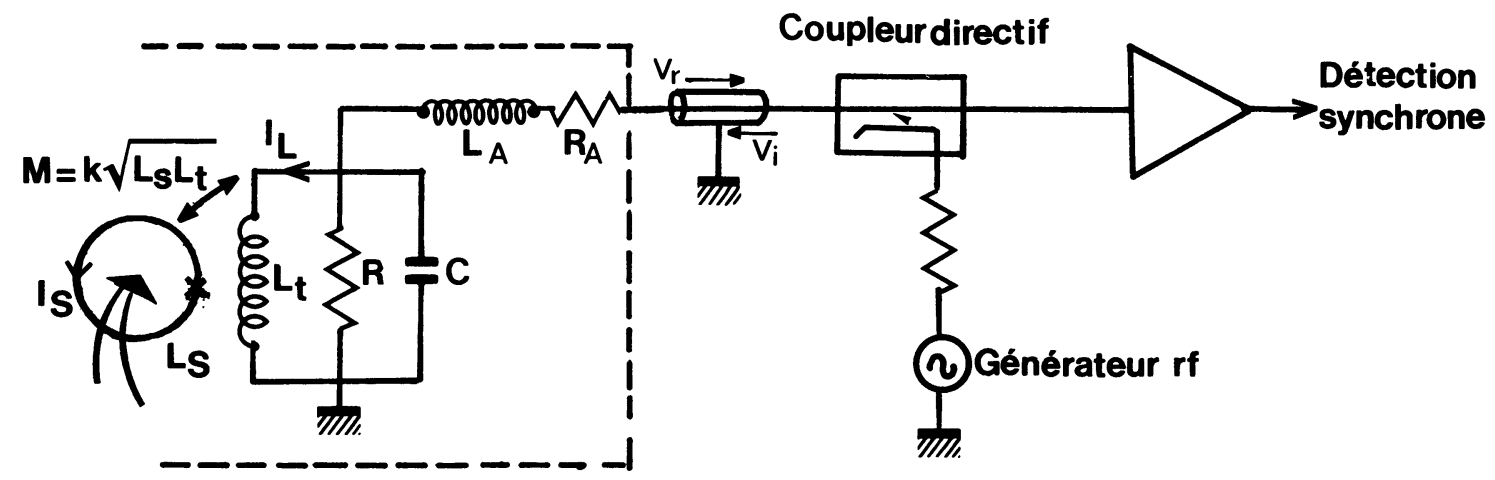

Fig. 1. - Schéma de la sonde adaptée. Le coupleur directif permet l'injection de l'onde radiofréquence incidente $V_{\mathrm{i}}$ et la séparation de l'onde réfléchie $V_{\mathrm{r}}$ vers l'amplificateur et la détection synchrone.

[Matched configuration assembly : the directional coupler carries out the feeding of the probe with the incident voltage wave $V_{\mathrm{i}}$ and selects the reflected wave $V_{\mathrm{r}}$ which is amplified and demodulated synchronously.]

que $\phi_{\mathrm{dc}}$ du SQUID. Une détection synchrone à la fréquence de pompe restitue aisément cette information. En régime bouclé, la contre-réaction de flux peut s'opérer simplement, via la ligne de transmission, par injection de courant dans la bobine radiofréquence.

$\mathrm{Si}$, en réflectométrie, la fonction de transfert en boucle ouverte $V_{\mathrm{S}}\left(\phi_{\mathrm{dc}}\right)$ admet toujours pour période le quantum de flux $\phi_{0}$, la recherche et l'interprétation théorique correcte des conditions de ce transfert restent jusqu'ici largement inexplorées ; nous tentons ici de combler cette lacune. En première partie une étude complémentaire du SQUID rf en configuration classique non bruyante est développée. Elle fait appel à l'analyse harmonique dont la portée, de prime abord restreinte, est étendue par une analyse temporelle valide quelle que soit l'intensité du couplage magnétique entre le SQUID et le circuit oscillant. Les résultats de ces analyses permettent de décrire le fonctionnement en réflectométrie. Les caractéristiques en phase et en quadrature de l'onde de tension réfléchie $V_{\mathrm{r}}$ en fonction de l'onde incidente $V_{\mathrm{i}}$, c'est-à-dire la réponse de la sonde adaptée, sont calculées dans l'hypothèse d'un système non bruyant.

En seconde partie nous précisons les effets associés à la présence du bruit de fond. La modification des grandeurs par effets non linéaires est alors abordée ; nous montrons qu'en ce domaine la source de bruit localisée au niveau du micropont de Dayem provoque l'essentiel de la déformation. Par suite, bien que ne connaissant pas la dépendance théorique de la probabilité de transition entre états de flux total du SQUID à micropont, nous en déduisons la valeur de la densité spectrale des fluctuations de nature intrinsèque qui est bien inférieure à la densité spectrale du bruit associé à l'électronique de détection placée à température ambiante. En dernier lieu les problèmes d'amélioration du rapport signal sur bruit sont envisagés pour ce système.

\section{Analyses du fonctionnement idéal des systèmes} classiques et adaptés.

2.1 ANALYSE HARMONIQUE. - Rappelons brièvement le principe des systèmes à SQUID rf. Le traitement semiclassique de l'anneau supraconducteur d'inductance $L_{\mathrm{S}}$, interrompu par une jonction Josephson de courant critique $I_{\mathrm{c}}$ et de résistance normale $R_{\mathrm{N}}$, introduit très naturellement [3,4], la caractéristique de flux magnétique $\phi_{\mathrm{X}}$ appliqué à l'anneau. La figure 2 montre que $\phi_{\mathrm{T}}\left(\phi_{\mathrm{X}}\right)$ est multivaluée, donc hystérétique, pour :

$$
\beta=2 \pi L_{\mathrm{S}} I_{\mathrm{c}} / \phi_{0}>1 .
$$

De ce fait, on comprend aisément le mode d'action du SQUID rf pour des fréquences de pompe très inférieures à $R_{\mathrm{N}} / L_{\mathrm{S}}$ lorsqu'un flux radiofréquence $\phi_{\text {rf }}$ et un flux quasistatique $\phi_{\mathrm{dc}}$ y sont conjointement appliqués. La réponse du système entier est subordonnée au nombre moyen de cycles d'hystérésis englobés par période rf ainsi qu'à la répartition temporelle des sauts de flux du SQUID pendant la période radiofréquence. Ces deux effets, associés respectivement aux processus de dissipation et de dispersion de l'énergie du champ radiofréquence au niveau du SQUID, sont fortement dépendants des flux $\phi_{\mathrm{dc}}$ et $\phi_{\mathrm{rf}}$ appliqués. L'analyse par la méthode de la balance harmonique permet de les traduire en termes d'impédances équivalentes à la fréquence $f_{\mathrm{P}}$ du champ radiofréquence.

La première étape du calcul consiste à établir le schéma équivalent du SQUID couplé magnétiquement à la bobine radiofréquence d'inductance $L_{\mathrm{t}}$. Celle-ci est traversée par un courant $I_{\mathrm{L}}$ développant la tension $V$ à ses bornes (les notations sont précisées en Fig. 3a). Afin que les grandeurs $I_{\mathrm{L}}$ et $V$ soient considérées comme quasisinusoïdales à $f_{\mathrm{P}}$, le coefficient de couplage magnétique $k$ est supposé petit devant l'unité. Choisissant comme origine des phases celle du courant $I_{\mathrm{L}}$, nous pouvons 


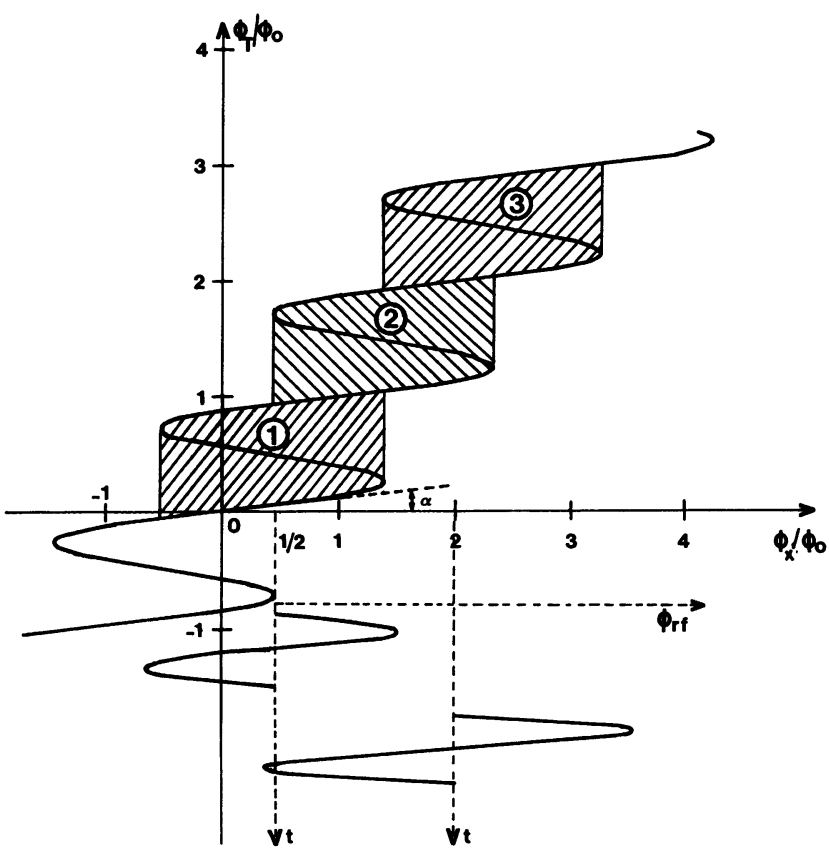

Fig. 2. - Flux total $\phi_{\mathrm{T}}$ en fonction du flux appliqué $\phi_{\mathrm{X}}$. Soumis à l'excitation $\phi_{\mathrm{X}}=\phi_{\mathrm{dc}}+\phi_{\mathrm{rf}} \cos \left(\omega_{\mathrm{P}} t\right)$ le cycle 1 est parcouru à chaque période radiofréquence si $\phi_{\mathrm{dc}}=\phi_{0} / 2$ alors que les cycles 2 et 3 le sont pour $\phi_{\mathrm{dc}}=2 \phi_{0}$.

[Total flux $\phi_{\mathrm{T}}$ of the SQUID versus the applied flux $\phi_{\mathrm{X}}$. Under application of a flux $\phi_{\mathrm{X}}=\phi_{\mathrm{dc}}+\phi_{\mathrm{rf}} \cos \left(\omega_{\mathrm{P}} t\right)$ hysteresis loop 1 is enclosed during each radiofrequency period if $\phi_{\mathrm{dc}}=\phi_{0} / 2$, for $\phi_{\mathrm{dc}}=2 \phi_{0}$ loops 2 and 3 are enclosed.]

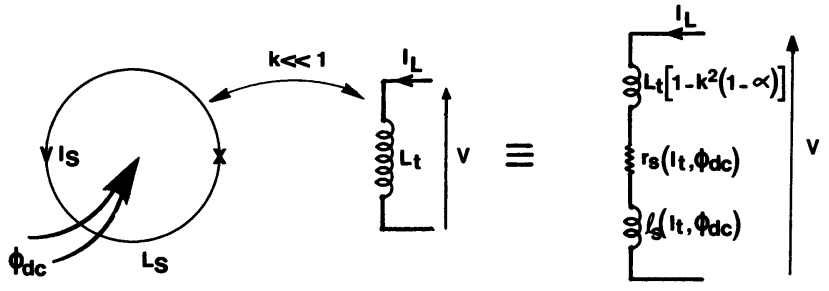

Fig. 3a - Circuit équivalent radiofréquence d'impédance $Z_{\mathrm{s}}$ du SQUID couplé à la bobine rf et notations correspondantes.

[Equivalent impedance $Z_{\mathrm{S}}$ of the SQUID coupled to the rf coil and notations.]

exprimer les composantes du courant $I_{\mathrm{S}}$ parcourant l'anneau, en phase et en quadrature avec le courant $I_{\mathrm{L}}$, en fonction de l'amplitude $I_{\mathrm{t}}$ de $I_{\mathrm{L}}$ et du flux quasistatique appliqué au SQUID. L'amplitude complexe de la tension $V$ s'exprime aisément en fonction des composantes de $I_{\mathrm{S}}$, nous pouvons donc déterminer à la pulsation :

$$
\omega_{\mathrm{P}}=2 \pi f_{\mathrm{P}},
$$

une impédance équivalente $Z_{\mathrm{S}}$ du SQUID couplé à l'inductance $L_{\mathrm{t}}$. Celle-ci se présente sous la forme suivante :

$$
\begin{aligned}
Z_{\mathrm{S}}=r_{\mathrm{S}}\left(I_{\mathrm{t}}, \phi_{\mathrm{dc}}\right)+ & j l_{\mathrm{S}}\left(I_{\mathrm{t}}, \phi_{\mathrm{dc}}\right) \omega_{\mathrm{P}}+ \\
& +j L_{\mathrm{t}}\left(1-k^{2}(1-\alpha)\right) \omega_{\mathrm{P}}
\end{aligned}
$$

où $r_{\mathrm{S}}\left(I_{\mathrm{t}}, \phi_{\mathrm{dc}}\right)$ et $l_{\mathrm{S}}\left(I_{\mathrm{t}}, \phi_{\mathrm{dc}}\right)$ sont des fonctions non linéaires, discontinues de l'amplitude du courant radiofréquence traversant l'inductance $L_{\mathrm{t}}$ et du flux quasistatique appliqué $\phi_{\mathrm{dc}}$. Le facteur correctif $\alpha$ est associé à la linéarisation de la caractéristique de flux du SQUID de la figure 2. Les fonctions $r_{\mathrm{S}}\left(I_{\mathrm{t}}, \phi_{\mathrm{dc}}\right)$ et $l_{\mathrm{S}}\left(I_{\mathrm{t}}, \phi_{\mathrm{dc}}\right)$ qui traduisent les effets non linéaires dispersifs et dissipatifs du SQUID, ont les allures typiques représentées en figure $3 b$ et $3 c$ pour
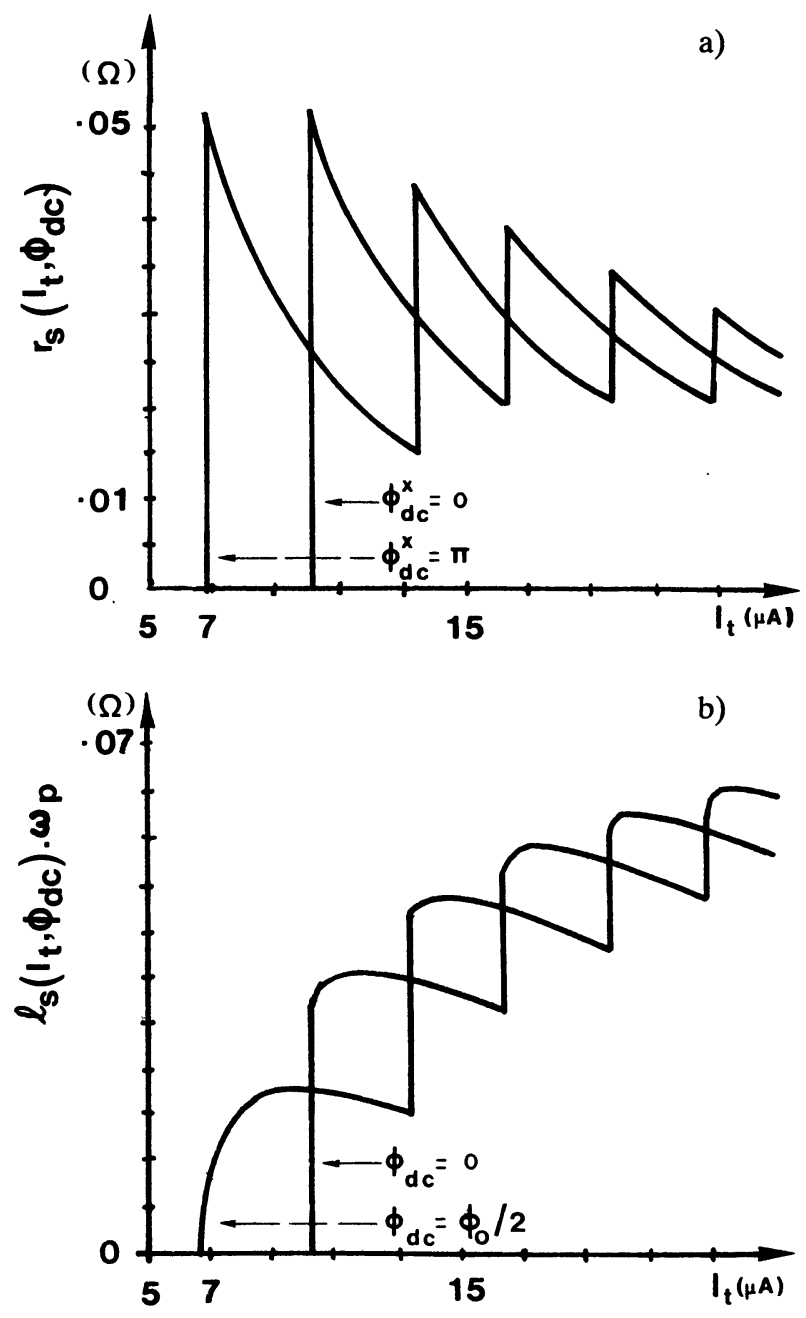

Figs. 3 b et 3 c. - Enveloppes des parties réelles $(3 \mathrm{~b})$ et imaginaires $(3 \mathrm{c})$ de la composante non linéaire de $Z_{\mathrm{S}}$ en fonction du flux $\phi_{\mathrm{dc}}$ et de l'amplitude $I_{\mathrm{t}}$ du courant radiofréquence. Les valeurs des paramètres sont : $L_{\mathrm{S}}=$ $5 \mathrm{nH} ; I_{\mathrm{C}}=5 \mu \mathrm{A} ; L_{\mathrm{t}}=205 \mathrm{nH} ; k=0,034 ; \phi_{\mathrm{dc}}=0$ ou $\phi_{0} / 2$.

[Real (3 b) and imaginary parts $(3 \mathrm{c})$ of the nonlinear component of $Z_{\mathrm{s}}\left(I_{\mathrm{t}}\right)$ for $\phi_{\mathrm{dc}}=0$ or $\phi_{0} / 2$ and the set of parameters indicated above.] 
des flux quasistatiques égaux à $n \phi_{0}$ et $(n+1 / 2) \phi_{0}$ ( $n$ entier). Ces résultats sont consistants avec ceux donnés par Silver et Zimmerman [5].

Partant du traitement précédent, le circuit classique RLC couplé au SQUID s'interprète comme un circuit oscillant dont l'inductance est complexe et présente l'impédance $Z_{S}\left(I_{t}, \phi_{\mathrm{dc}}\right)$ à $\omega_{\mathrm{p}}$. Un calcul linéaire point par point permet alors de reconstituer par morceaux (Fig. 4) la caractéristique de tension $V_{\text {rf }}$ développée aux bornes du circuit oscillant en fonction de l'amplitude $I_{\mathrm{rf}}$ du courant injecté.

Or, si pour les régions quasilinéaires de forte pente le nombre de cycles d'hystérésis englobé par période rf est entier et ne varie pas avec la valeur du courant $I_{\mathrm{rf}}$, en revanche, pour les régions de faible pente le nombre "moyen" de cycles d'hystérésis englobé par le SQUID varie continûment d'une valeur entière $N$ jusqu'à la valeur $N+1$ ou $N+2$. Ainsi, la figure 4 , où sont reportées les allures typiques de $V_{\mathrm{rf}}$ résultant du traitement précédent, nous montre que la méthode harmonique est insuffisante. Pour la sonde classique, seules les régions de fortes pentes sont bien décrites et ce uniquement pour des fréquences de pompe supérieures à la fréquence de résonance $f_{\mathrm{t}}$ du circuit RLC couplé au SQUID :

$$
f_{\mathrm{t}}=\frac{1}{2 \pi}\left\{L_{\mathrm{t}} C\left[1-k^{2}(1-\alpha)\right]\right\}^{-1 / 2} .
$$

Pour les fréquences inférieures à $f_{\mathfrak{t}}$, la tendance inductive de $Z_{\mathrm{S}}$ liée au mécanisme de dispersion de l'énergie rf tend à ramener le circuit vers la résonance, et le calcul point par point à partir de la valeur de $Z_{\mathrm{s}}$ conduit à des rebroussements de $I_{\text {rf }}$ (Fig. 4) ce qui n'est pas correct puisque dans la

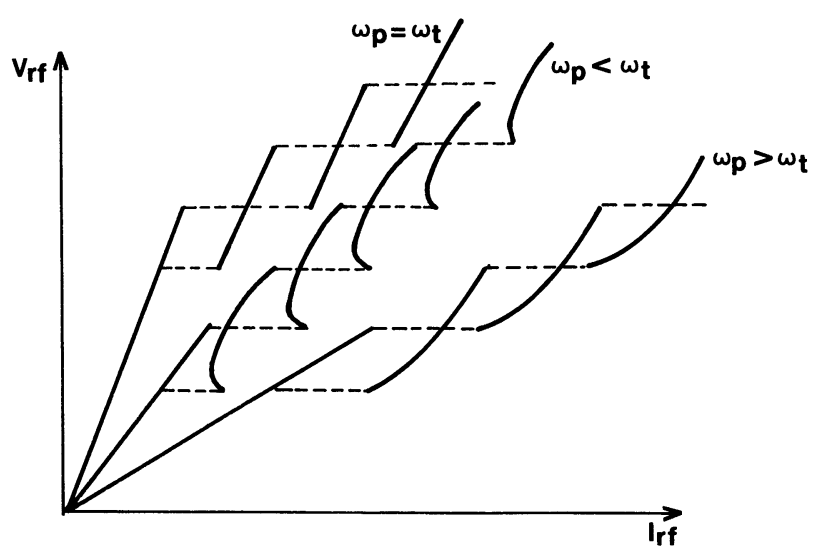

Fig. 4. - Allures typiques des caractéristiques de tension $V_{\mathrm{rf}}-I_{\mathrm{rf}}$ obtenues par la méthode de la balance harmonique pour des pulsations de pompe $\omega_{\mathrm{P}}$ voisines de la pulsation de résonance du circuit bouchon.

[Typical staircase pattern $V_{\mathrm{rf}}-I_{\mathrm{rf}}$ obtained by the harmonic balanced method for pumping angular frequencies close to the tank circuit tuning.] pratique ce courant est imposé. Par ailleurs, la structure fine des plateaux de tension ne peut être décrite puisque le calcul harmonique de $Z_{\mathrm{S}}$ ne fait en aucun cas intervenir la notion de nombre « moyen » de cycles d'hystérésis parcourus par période radiofréquence. La description par balance harmonique du SQUID rf est donc incomplète et nous noüs proposons maintenant de la compléter par une analyse temporelle.

2.2 ANAlyse temporelle. - Par la suite, nous supposons la durée de la période radiofréquence très supérieure à la constante de temps $L_{\mathrm{S}} / R_{\mathrm{N}} \mathrm{du}$ SQUID.

2.2.1 Mise en équation et solutions. - Une alternative à la description du SQUID rf au moyen de sa caractéristique de flux résulte de l'écriture du courant $I_{S}$ circulant dans l'anneau en fonction du courant $I_{\mathrm{L}}$ dans la bobine radiofréquence et du flux magnétique appliqué $\phi_{\mathrm{dc}}$ :

$$
I_{\mathrm{S}}=\frac{\alpha-1}{L_{\mathrm{S}}}\left(M I_{\mathrm{L}}+\phi_{\mathrm{dc}}+f\left(I_{\mathrm{S}}\right) \phi_{0}\right),
$$

où $M=k \sqrt{L_{\mathrm{S}} L_{\mathrm{t}}}$ est la mutuelle inductance entre l'anneau et la bobine rf, $\alpha$ est défini en (2.3) et $f\left(I_{\mathrm{S}}\right)$ est une fonction à valeurs entières définie comme suit :

- $f\left(I_{\mathrm{S}}\right)$ admet au moins une valeur entière $N$ telle que la valeur absolue de $I_{\mathrm{S}}(2.5)$ soit inférieure au courant critique $I_{\mathrm{c}}$ de la jonction ;

- $f\left(I_{\mathrm{S}}\right)$ s'accroît d'une unité si, à $\mathrm{d} I_{\mathrm{L}} / \mathrm{d} t$ positif, $I_{\mathrm{S}}$ atteint la valeur $-I_{\mathrm{c}}$ et décroît d'une unité si, à $\mathrm{d} I_{\mathrm{L}} / \mathrm{d} t$ négatif, $I_{\mathrm{S}}$ atteint la valeur $+I_{\mathrm{c}}$.

Dans ces conditions, l'amplitude du courant $I_{\mathrm{S}}$ du SQUID est bornée à la valeur $I_{\mathrm{c}}$. La figure 5 précise la dépendance en $I_{\mathrm{L}}$ ainsi que la nature

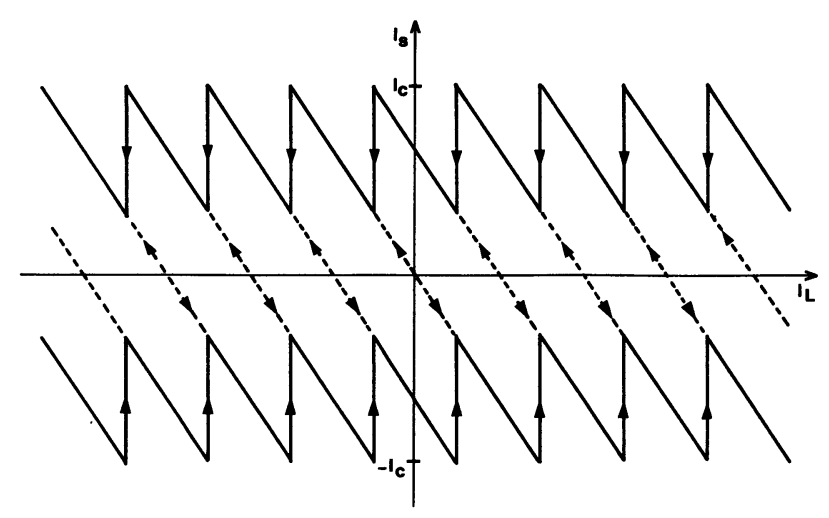

Fig. 5. - Tracé du courant $I_{\mathrm{S}}$ circulant dans l'anneau supraconducteur en fonction du courant $I_{\mathrm{L}}$ dans la bobine couplée. Le comportement est de nature hystérétique via les chemins tracés en pointillés.

[Plot of the ring current $I_{\mathrm{S}}$ versus the coil current $I_{\mathrm{L}}$. The dotted lines show the hysteretic behaviour.] 
«quasidiscontinue » de l'évolution temporelle de $I_{\mathrm{S}}$ à l'échelle de la période radiofréquence. Le circuit est représenté en figure 6 avec les notations utilisées. Le flux magnétique $\phi_{\mathrm{L}}$ traversant $L_{\mathrm{t}}$ s'écrit :

$$
\begin{aligned}
\phi_{\mathrm{L}}(t)=L_{\mathrm{t}}\left[1-k^{2}(1-\alpha)\right] I_{\mathrm{L}}(t)+ \\
+\frac{M(\alpha-1)}{L_{\mathrm{S}}}\left[\phi_{\mathrm{dc}}+\phi_{0} f\left(I_{\mathrm{S}}\right)\right] ;
\end{aligned}
$$

le courant d'attaque du circuit oscillant s'exprime par :

$I(t)=I_{\mathrm{L}}(t)+I_{\mathrm{R}}(t)+I_{\mathrm{c}}(t)=I_{\mathrm{rf}} \cos \left(\omega_{\mathrm{P}} t+\theta_{0}\right) ;$

et la tension à ses bornes par :

$V(t)=R I_{\mathrm{R}}(t)=\mathrm{d} \phi_{\mathrm{L}}(t) / \mathrm{d} t=r I_{\mathrm{c}}(t)+V_{\mathrm{c}}(t)$.

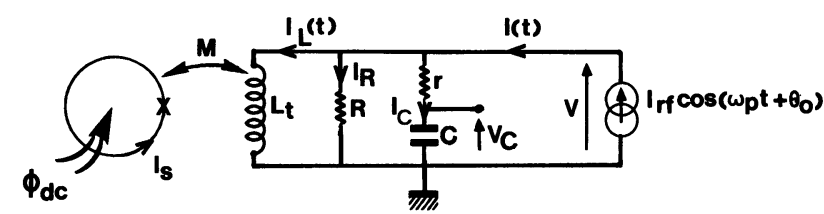

Fig. 6. - Circuit modèle et notations utilisés pour l'analyse temporelle du SQUID couplé au circuit oscillant.

[Pattern circuit and notations used to work out the time analysis of the SQUID coupled to the tank circuit.]

En exprimant $\phi_{\mathrm{L}}(t), I_{\mathrm{S}}(t)$ et $V_{\mathrm{c}}(t)$ en fonction du courant de pompe $I(t)$, du flux quasistatique $\phi_{\mathrm{dc}}$ et des valeurs initiales $\phi_{\mathrm{L}}(0)$ et $V_{\mathrm{c}}(0)$, puis en résolvant ces équations par l'intermédiaire de la transformée de Laplace, sur les domaines où la fonction $f\left(I_{\mathrm{S}}\right)$ précédemment définie est constante, on obtient aisément l'évolution temporelle de $\phi_{\mathrm{L}}, I_{\mathrm{S}}$ et $V_{\mathrm{c}}$ correspondant aux zones linéaires de la figure 5. Lorsque les conditions requises pour que $f\left(I_{\mathrm{S}}\right)$ varie d'une unité sont réunies à un instant $t_{0}$, les valeurs atteintes par $\phi_{\mathrm{L}}$ et $V_{\mathrm{c}}$ à $t=t_{0}$ sont prises comme valeurs initiales du système d'équation modifié pour le segment temporel suivant par la transition (saut de flux total du SQUID) :

$$
f\left(I_{\mathrm{S}}, t>t_{0}\right)=f\left(I_{\mathrm{S}}, t<t_{0}\right) \pm 1 .
$$

et valables jusqu'à ce qu'une nouvelle transition intervienne. En poursuivant ce calcul sur un grand nombre de périodes rf (très supérieur au coefficient de qualité $Q$ du circuit oscillant), on obtient la réponse du système pour un courant radiofréquence $I_{\mathrm{rf}}$ et un flux quasistatique $\phi_{\mathrm{dc}}$ imposé.

2.2.2 Apports de l'analyse temporelle. - Cette méthode permet d'analyser la réponse du système à l'excitation en courant $I_{\mathrm{rf}}$ quel que soit le couplage $k$ anneau-bobine rf et sans restrictions des valeurs de $Q$ ni de la pulsation d'attaque $\omega_{\mathrm{p}}$. Les figures $7 \mathrm{a}-7 \mathrm{~b}$

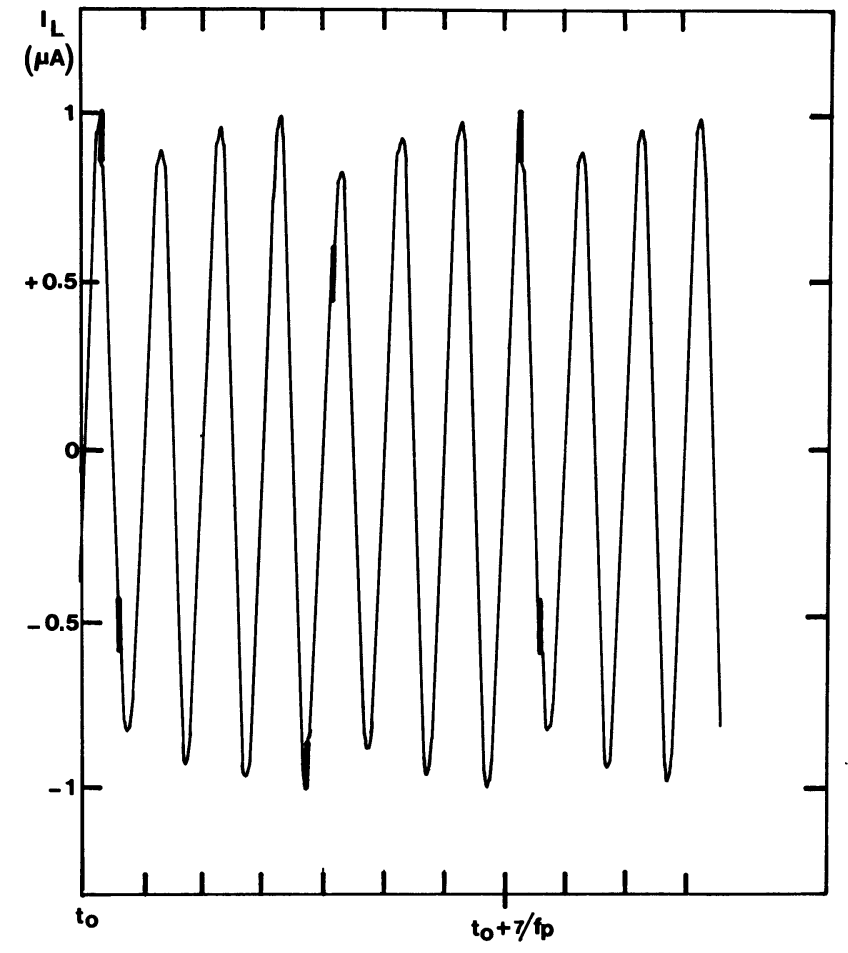

a)

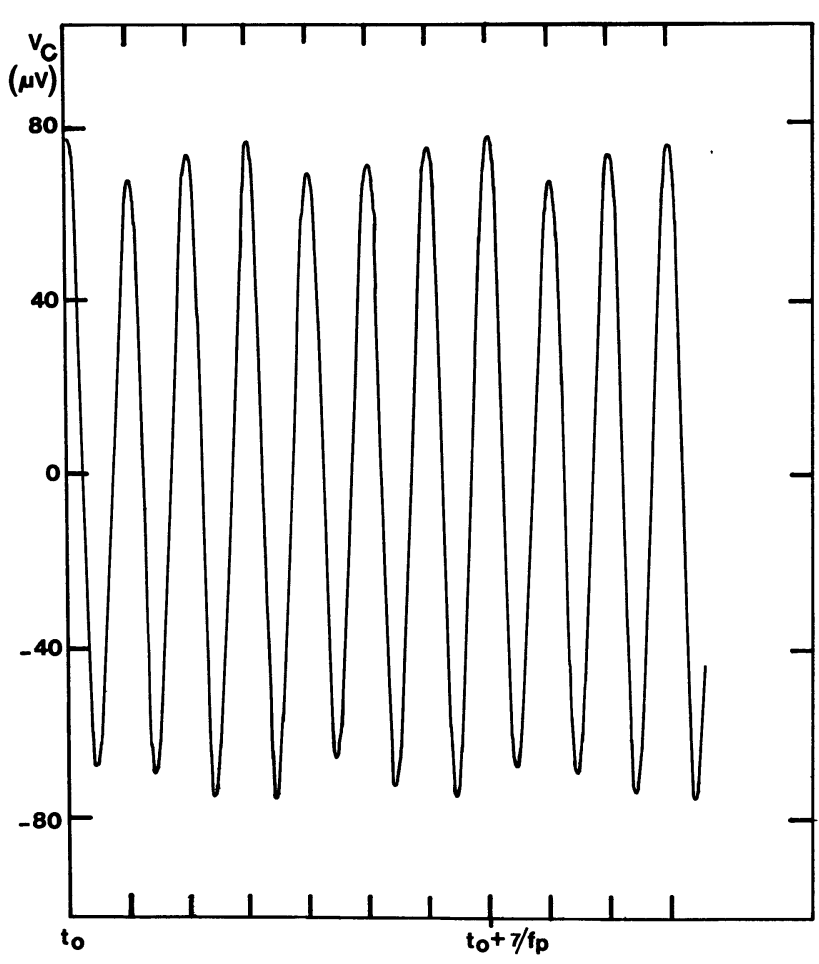

b)

Figs. 7 a et 7 b. - Evolution temporelle du courant $I_{\mathrm{L}}$ dans la bobine rf $(7 \mathrm{a})$ et de la tension $V_{\mathrm{c}}(7 \mathrm{~b})$ pour les paramètres suivants : $I_{\mathrm{C}}=10 \mu \mathrm{A}, L_{\mathrm{S}}=0,5 \mathrm{nH} ; k=0,5$; $\phi_{\mathrm{dc}}=0 ; L_{\mathrm{t}}=200 \mathrm{nH} ; C=25 \mathrm{pF} ; Q=4 ; r \rightarrow 0 ; f_{\mathrm{P}}=$ $82,187 \mathrm{MHz} ; I_{\mathrm{rf}}=0,255 \mu \mathrm{A}$. La période des oscillations de relaxation est alors de $7 / f_{\mathrm{P}}$.

[Coil current $I_{\mathrm{L}}(7 \mathrm{a})$ and voltage $V_{\mathrm{c}}(7 \mathrm{~b})$ time evolutions for the previous set of parameters. The period of the relaxations mechanisms is $7 / f_{\mathrm{P}}$.] 
illustrent le cas du fort couplage et mettent en exergue les processus de relaxation du circuit oscillant. Des oscillations périodiques de relaxation sont d'ailleurs systématiques lorsque $\omega_{\mathrm{p}}>\omega_{\mathrm{t}}$. Il est possible dans ces conditions de décomposer $V_{\mathrm{c}}(t)$ suivant ses composantes en phase et en quadrature avec le courant de pompe $I_{\text {rf }}$ et de calculer la valeur moyenne de leur amplitude sur une période de relaxation. Un tel calcul permet de déterminer en particulier la structure fine des plateaux de la courbe $V_{\mathrm{rf}}\left(I_{\mathrm{rf}}\right)$ et donc de déduire l'impédance équivalente $Z_{S}^{\prime}\left(I_{\text {rf }}\right)$ du SQUID couplé à la bobine rf quelle que soit l'amplitude du courant $I_{\mathrm{rf}}$. Cette procédure a été menée à son terme et a montré la concordance avec l'analyse harmonique dans les plages de validité de cette dernière. Il faut cependant remarquer que les calculs sur ordinateur sont longs, surtout pour des coefficients $Q$ élevés, alors que l'analyse harmonique conduit à un calcul plus court et indépendant de la valeur de $Q$. C'est la raison pour laquelle l'analyse temporelle ne nous a servi qu'à préciser la structure fine des plateaux de tension et l'impédance $Z_{\mathrm{S}}^{\prime}\left(I_{\mathrm{rf}}\right)$ correspondante. En réflectométrie, les conditions expérimentales se traduisant par $Q \gg 1, \omega_{\mathrm{p}}>\omega_{\mathrm{t}}$, aussi l'analyse harmonique est en partie applicable à

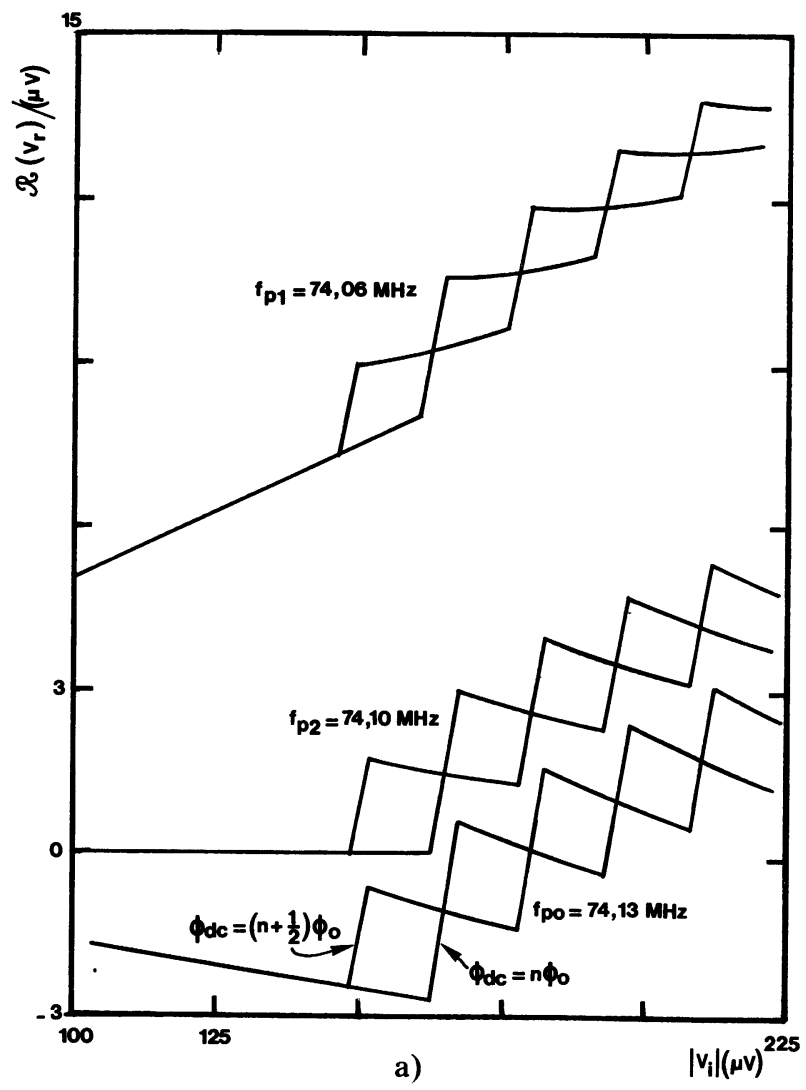

Figs. 8 a et 8 b. - Tracé des enveloppes à $\phi_{\mathrm{dc}}=$ $n \phi_{0}$ et $(n+1 / 2) \phi_{0}$ des parties réelle $(8 \mathrm{a})$ et imaginaire $(8 \mathrm{~b})$ de l'onde de tension réfléchie $V_{\mathrm{r}}$ en fonction de l'onde incidente $V_{\mathrm{i}}$ dans le cas idéal non bruyant. A la résonance $f_{\mathrm{P} 0}$ l'impédance de la sonde est de $48.4 \Omega$. condition de la compléter par les procédures de linéarisation adéquates au voisinage des discontinuités qu'elle présente.

\subsection{CONDITIONS IDÉALES DE TRANSFERT} « $V_{\mathrm{r}}-V_{\mathrm{i}} »$. - L'examen de l'impédance totale de la sonde adaptée (Fig. 1) permet d'établir l'expression générale des pulsations de résonance. Dans les conditions habituelles de fonctionnement - coefficient de qualité du circuit oscillant isolé élevé $(Q \gg 1)$ et pertes de la bobine d'adaptation faibles $\left(R_{\mathrm{A}} \ll L_{\mathrm{A}} \omega_{\mathrm{P}}\right)$ - ces pulsations de résonance $\omega_{\mathrm{t}}$ et $\omega_{\mathrm{r}}$ respectivement associées aux valeurs haute et basse de l'impédance de la sonde, sont données avec une bonne approximation par:

$$
\omega_{\mathrm{t}}=\left[L_{\mathrm{t}} C\left(1-k^{2}(1-\alpha)\right)\right]^{-1 / 2}
$$

et

$$
\omega_{\mathrm{r}}=\omega_{\mathrm{t}}\left(1+L_{\mathrm{t}} / L_{\mathrm{A}}\right)^{1 / 2}
$$

$Z_{\mathrm{r}}$ l'impédance totale de la sonde s'écrit pour $\omega_{\mathrm{P}}=\omega_{\mathrm{r}}$ :

$$
Z_{\mathrm{r}}=\left(1+L_{\mathrm{t}} / L_{\mathrm{A}}\right) \frac{L_{\mathrm{A}}^{2} \omega_{\mathrm{t}}}{Q L_{\mathrm{t}}}
$$

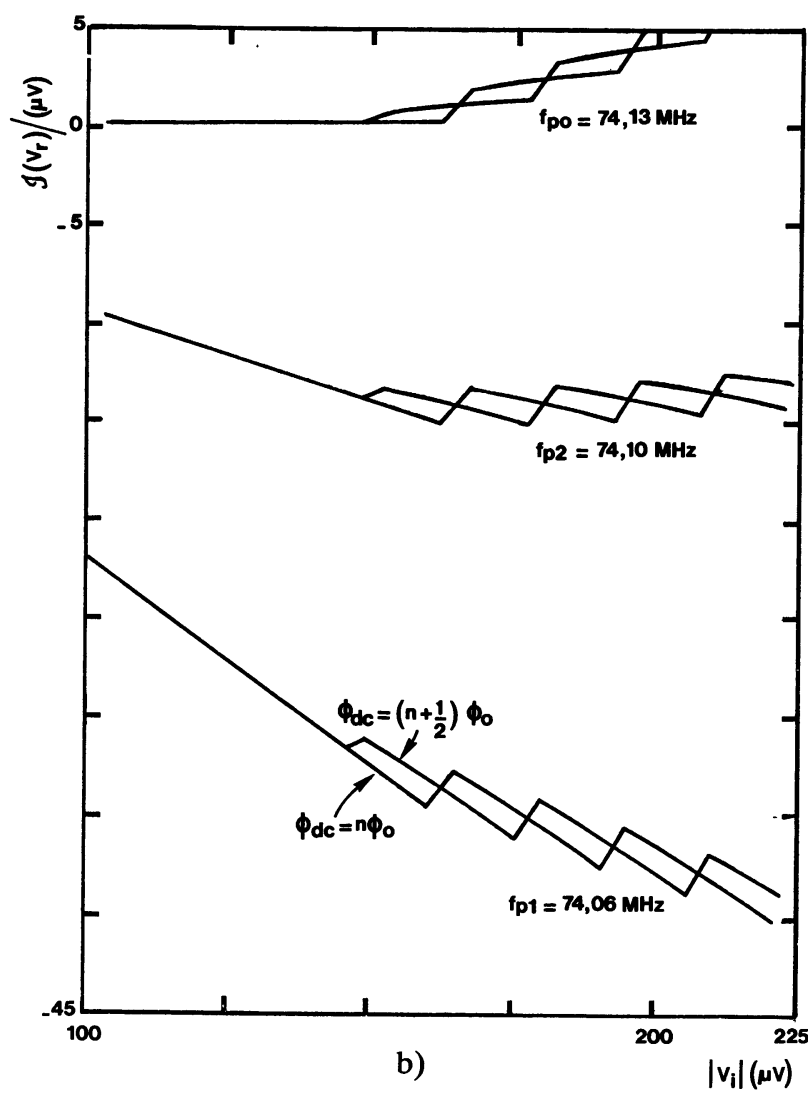

[Real and imaginary parts of the reflected wave $V_{\mathrm{r}}$ versus the incident wave $V_{\mathrm{i}}$ for the flux values $\phi_{\mathrm{dc}}=\boldsymbol{n} \phi_{0}$ and $(n+1 / 2) \phi_{0}$. At the pumping frequency $f_{\mathrm{P} 0}$ the probe impedance is $48.4 \Omega$.] 
L'adaptation d'impédance de la sonde à la ligne de transmission est donc possible au voisinage de $\omega_{\mathrm{r}}$ par action sur la valeur de $L_{\mathrm{A}}$. Puisque $\omega_{\mathrm{r}}>\omega_{\mathrm{t}}$ (Eq. (2.11)) la discussion concernant l'utilisation de l'analyse harmonique est directement justifiée. Les figures $8 \mathrm{a}$ et $8 \mathrm{~b}$ représentent un tracé des caractéristiques idéales des composantes en phase et en quadrature de la tension réfléchie $V_{\mathrm{r}}$ avec la tension incidente $V_{\text {i }}$ pour les valeurs $n \phi_{0}$ et $(n+1 / 2) \phi_{0}$ du flux quasistatique $\phi_{\mathrm{dc}}$ appliqué au SQUID. Ces tracés idéaux sont naturellement affectés par l'action du bruit de fond du système dont les effets sont maintenant abordés.

\section{Comportement de la sonde adaptée en présence de bruit.}

3.1 DÉFORMATIONS APPORTÉES AUX CARACTÉRISTIQUES IDÉALES. - Les caractéristiques statiques réelles expérimentales présentées en figures $9 a$ et $9 b$ diffèrent des caractéristiques idéales des figures $8 \mathrm{a}$ et $8 \mathrm{~b}$. Cet écart est dû au bruit de fond et aux nonlinéarités du système, l'objet de ce paragraphe est de le caractériser plus précisément.

3.1.1 Déformations occasionnées par les sources de bruit externes au SQUID. - Nous admettons dans notre analyse que jusqu'à environ $500 \mathrm{MHz}$ le circuit équivalent de la sonde est bien connu. Le bruit provient essentiellement de l'électronique d'injection et de préamplification à température ambiante ; il se traduit au niveau de la bobine if couplée au SQUID par un courant de bruit de densité spectrale $S_{\mathrm{i}}(f)$ en bande étroite autour de $\omega_{\mathrm{r}}$. Le courant $I_{\mathrm{L}}$ est modulé en amplitude et en phase, de façon aléatoire mais lentement variable devant sa composante à $\omega_{\mathrm{P}}[6]$. Pour un rapport signal sur bruit suffisant, l'enveloppe de l'amplitude $I_{\mathrm{t}}$ du courant $I_{\mathrm{L}}$ est décrite par une densité de probabilité gaussienne de déviation standard $\sigma_{\mathrm{t}}$ :

$$
P_{\mathrm{G}}\left(I_{\mathrm{t}}\right)=\frac{1}{\sqrt{2 \pi} \sigma_{\mathrm{t}}} \exp \left[-\left(\frac{I_{\mathrm{t}}-\left\langle I_{\mathrm{t}}\right\rangle}{\sqrt{2} \sigma_{\mathrm{t}}}\right)^{2}\right] \text {, }
$$

où $\left\langle I_{t}\right\rangle$ est la valeur moyenne de l'amplitude du courant rf dans la self-inductance $L_{\mathrm{t}}$.

Figs. 9 a et 9 b. - Tracés expérimentaux correspondant aux courbes précédentes 8 a et $8 \mathrm{~b}$. Le bruit arrondit considérablement les caractéristiques idéales par effet des non-linéarités apportées par le SQUID.

[Experimental plots of the reflected wave $V_{\mathrm{r}}$ versus the incident one. The noise softens the characteristics shown in figures $8 \mathrm{a}$ and $8 \mathrm{~b}$ by the ways of the SQUID nonlinearities. The parameter set is the following :

$L_{\mathrm{S}}=0.5 \mathrm{nH} ; \quad I_{\mathrm{c}}=22 \mu \mathrm{A} ; \quad \alpha=0.15 ; \quad L_{\mathrm{t}}=205 \mathrm{nH} ;$ $Q=250 ; \quad C=25 \mathrm{pF} ; \quad k=0.036 ; \quad L_{\mathrm{A}}=2.162 \mu \mathrm{H}$; $R_{\mathrm{A}}=4.3 \Omega ; \quad f_{\mathrm{P} 0}=74.130 \mathrm{MHz} ; \quad f_{\mathrm{P} 1}=74.06 \mathrm{MHz}$; $f_{\mathrm{P} 2}=74.1 \mathrm{MHz}$.]

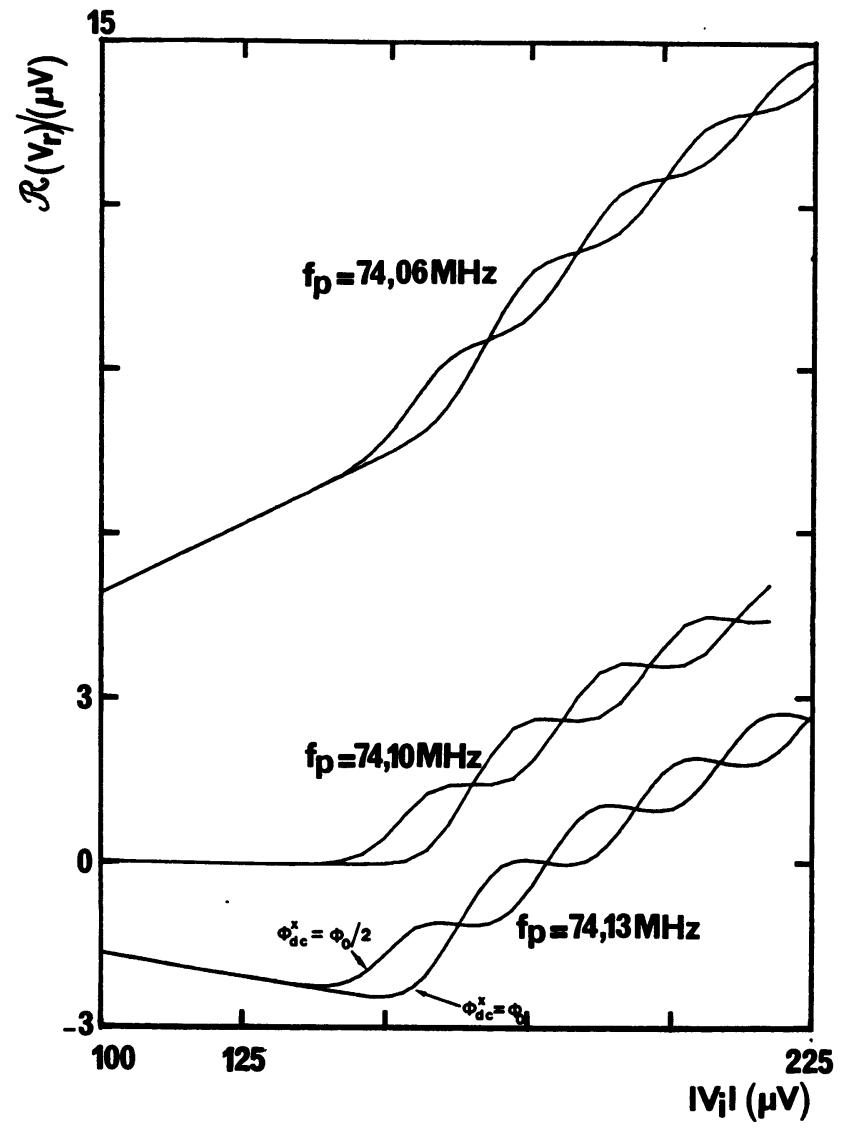

a)

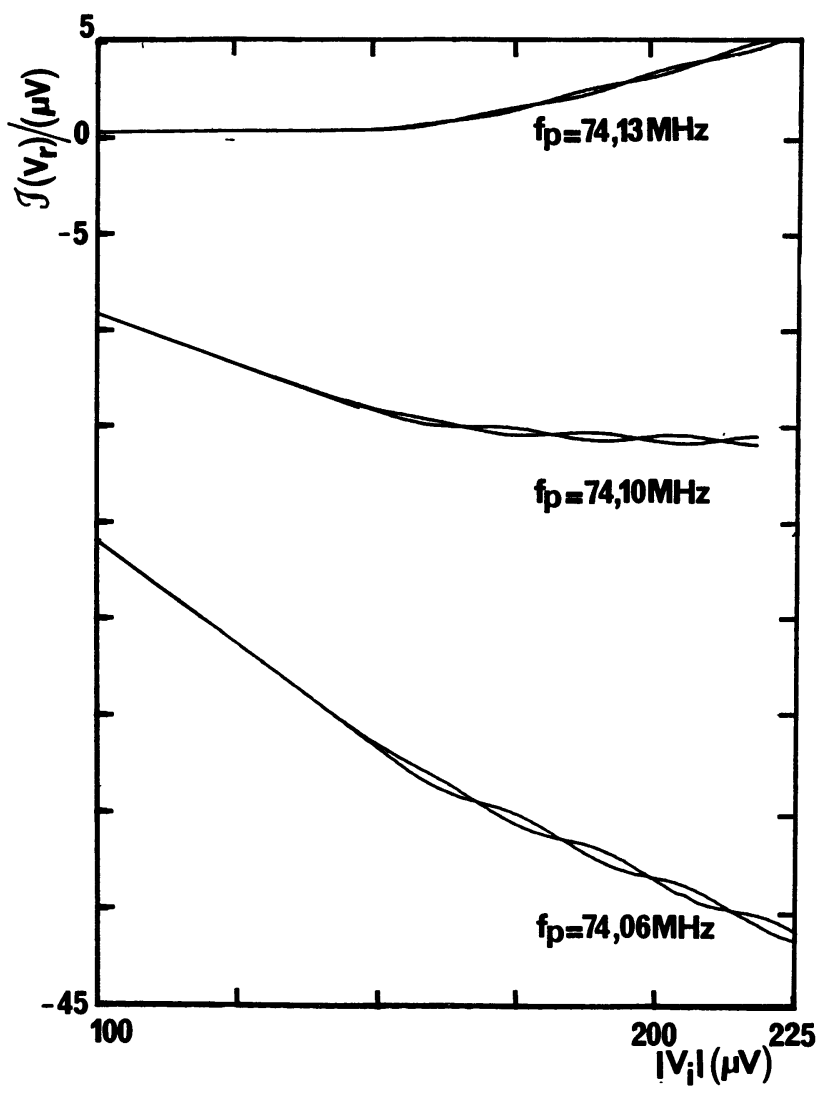

b) 
Le calcul de la réponse de la sonde en présence de ce bruit est effectué de la manière suivante : pour une injection de courant dans la bobine rf d'amplitude moyenne $\left\langle I_{t}\right\rangle$ nous considérons une infinité de sous-systèmes identiques dont le courant $I_{\mathrm{L}}$ est sinusoïdal d'amplitude $I_{\mathrm{t}}$ distribuée autour de $\left\langle I_{t}\right\rangle$. La réponse de la sonde en présence de bruit est ensuite déterminée en calculant la moyenne pondérée par $P_{\mathrm{G}}\left(I_{\mathrm{t}}\right)$ des réponses idéales de chaque sous-système. Compte tenu du bruit de l'électronique associée, le calcul de la densité spectrale $S_{\mathrm{i}}(f)$ de $I_{\mathrm{L}}$ peut être mené [7]. La valeur de l'écart quadratique moyen $\sigma_{t}$ correspondant pour nos caractéristiques expérimentales est de $0,02 \phi_{0} / M$ où $M$ est la mutuelle inductance entre le SQUID et la bobine radiofréquence. Par contre la valeur de $\sigma_{\mathrm{t}}$ qui conviendrait pour reproduire au mieux la déformation des caractéristiques expérimentales est d'environ un ordre de grandeur plus élevée. Les sources de bruit externes au SQUID n'interviennent donc que très peu dans la déformation des caractéristiques idéales; les causes doivent être recherchées ailleurs notamment au niveau du micropont du SQUID dont la résistance normale constitue la source de bruit intrinsèque.

\subsubsection{Déformations occasionnées par le bruit propre} $d u$ SQUID. - La relation courant-phase des microponts diffère notablement de la relation purement sinusoïdale des jonctions Josephson tunnel comme l'ont clairement montré Pascal [8] ou Likharev [9]. Ainsi, les méthodes et résultats de J. Kurkijärvi [10, 11], de K. K. Likharev [12] et de Danilov [13] ne sont guère applicables pour prévoir les déformations et fluctuations d'origine intrinsèque puisque, à notre connaissance, l'expression théorique de la probabilité de transition entre états de flux total du SQUID à micropont de Dayem n'est pas clairement établie. Nous ne pouvons donc analyser précisément les modifications apportées aux valeurs moyennes des grandeurs électriques de la sonde en présence de bruit intrinsèque du SQUID alors que nous avons montré au paragraphe précédent que ce bruit avait une influence prépondérante. Nous pouvons, en revanche, évaluer de façon précise les fluctuations autour des valeurs moyennes modifiées, qu'elles soient d'origine interne ou externe au SQUID; ce point est abordé au paragraphe suivant.

\subsection{ANAlyse Des fluctuAtions EN TERME DE BRUIT ÉQUIVALENT EN FLUX.}

3.2.1 Fluctuations intrinsèques. - Comme l'ont montré Likharev et Danilov [13] pour la configuration classique avec attaque en courant, les déformations des valeurs statiques sont fonction de la probabilité de transition $p$ d'un état de flux total du SQUID vers un état voisin, la transition s'effectuant aux extrêma du flux radiofréquence d'amplitude $\phi_{\text {rf }}$ appliqué au SQUID. Ces auteurs ont également montré pour les SQUID rf usuels largement hystérétiques $(\beta \gg 1)$ et faiblement couplés $(k \ll 1)$ que les fluctuations intrinsèques sont également liées à $p$ mais aussi à sa densité $\sigma$ :

$$
\sigma=\frac{\phi_{0}}{2 \pi} \frac{\mathrm{d} p}{\mathrm{~d} \phi_{\mathrm{rf}}}
$$

la densité spectrale $S_{\mathrm{i}}$ du bruit intrinsèque équivalent en flux s'écrivant :

$$
S_{\mathrm{i}}=\frac{\phi_{0}}{2 \pi}\left[\frac{p(1-p)}{\sigma^{2} \omega_{\mathrm{P}}}\right]^{1 / 2} .
$$

Par mesure de $p$ et $\sigma$ en un point de fonctionnement donné en utilisant la distorsion des caractéristiques, les fluctuations de flux associées peuvent en être déduites au moins pour le schéma classique de la sonde. Pour cette configuration, $\sigma$ est déduit de la caractéristique en plateau $V_{\mathrm{rf}}-I_{\mathrm{rf}}$ en effectuant l'opération $\mathrm{d} I_{\mathrm{rf}} / \mathrm{d} V_{\mathrm{rf}}$ le long du plateau à $\phi_{\mathrm{dc}}$ constant et en normalisant l'aire sous la courbe à l'unité ; $p$ est alors accessible par intégration. Cependant nous avons montré qu'une opération analogue peut s'effectuer à partir de la partie réelle de l'impédance $Z_{\mathrm{S}}\left(I_{\mathrm{t}}\right)$ définie antérieurement par l'équation (2.3) qui se déduit aisément de caractéristiques expérimentales de tension réfléchie $V_{\mathrm{r}}$ analogues à celles présentées en figures $9 \mathrm{a}$ et $9 \mathrm{~b}$. Par ailleurs Bélonogov et al. [14] et Likharev [12] ont étudié les bruits équivalents en flux pour les démodulations d'amplitude, de phase ou synchrone, et ont conclu que dans chaque cas la densité spectrale de bruit intrinsèque est donnée par l'équation (3.3).

L'analyse de la courbe de réponse du SQUID $V_{\mathrm{r}}=f\left(V_{\mathrm{i}}, \phi_{\mathrm{dc}}\right)$ pour $\phi_{\mathrm{dc}}=\phi_{0} / 4$, le point de repos habituel du SQUID en régime bouclé, permet de déduire la distribution de probabilité de transition $p$ et sa dérivée $\sigma$ illustrées en figure 10. Ces courbes donnent les valeurs expérimentales de la probabilité de transition $p$ et de sa densité $\sigma$ en fonction de l'amplitude réduite du flux $\phi_{\mathrm{rf}}$ radiofréquence appliqué. Pour le point de repos choisi on obtient $p=0,5$ et $\sigma=0,33$ et une évaluation précise de la densité de bruit intrinsèque de flux :

$$
S_{\mathrm{i}}=1,1 \times 10^{-5} \phi_{0} / \sqrt{\mathrm{Hz}} .
$$

Remarquons que la largeur à mi-hauteur de $\sigma\left(\phi_{\text {rf }}\right)$ obtenue est voisine de $\phi_{0} / 5$, ce qui représente environ 5 fois la largeur de la distribution évaluée d'après l'expression de Kurkijärvi [11]. Signalons pour terminer que l'évaluation de $p$ et $\sigma$ permet de retrouver le paramètre $\alpha$, défini par Jackel et Buhrman [15] relatif à la pente des plateaux de tension de la configuration classique qui, pour notre SQUID, aurait une valeur voisine de 0,5 . 


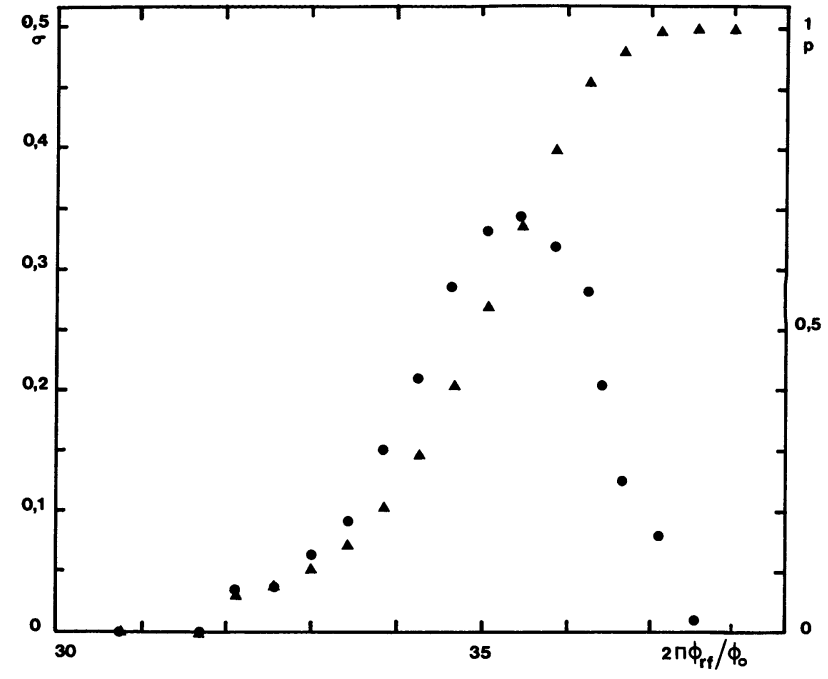

Fig. 10. - Evolution de la probabilité de transition $p(\Delta)$, intervenant à chaque période radiofréquence, et de la densité correspondante $\sigma=\frac{\phi_{0}}{2 \pi} \frac{\mathrm{d} p}{\mathrm{~d} \phi_{\mathrm{rf}}}(\bullet)$ en fonction du flux radiofréquence réduit appliqué à $\phi_{\mathrm{dc}}=\phi_{0} / 4$. Ces données sont déduites des caractéristiques expérimentales de tension réfléchie $V_{\mathrm{r}}$.

[Plot of the transition probability $p(\Delta)$ occurring at each rf period and associated density $\sigma=\frac{\phi_{0}}{2 \pi} \frac{\mathrm{d} p}{\mathrm{~d} \phi_{\mathrm{rf}}}(\bullet)$ as a function of the reduced applied flux $\left(\phi_{\mathrm{dc}}=\phi_{0} / 4\right)$. These data are deduced from the experimental characteristics of the reflected wave $V_{\mathrm{r}}$ ]

3.2.2 Fluctuations extrinsèques. - L'analyse' des sources de bruit - circuit oscillant à $4,2 \mathrm{~K}$, préamplificateur et impédance interne du générateur d'attaque à température ambiante - montre que le bruit du préamplificateur est le facteur dominant. Exprimé en terme de bruit équivalent en flux sa densité spectrale vaut pour nos conditions expérimentales $(*)$ :

$$
S_{\mathrm{e}}=10^{-4} \phi_{0} / \sqrt{\mathrm{Hz}},
$$

valeur très supérieure à la contribution intrinsèque.

3.3 RECHERCHE DES CONDITIONS OPTIMALES DE FONCTIONNEMENT DE LA SONDE ADAPTÉE. - Nos investigations numériques et expérimentales $[7,16$, 17] ont donné les résultats que nous résumons cidessous :

- La fréquence de pompe doit être égale à la fréquence de résonance série de la sonde $\omega_{\mathrm{r}}$ définie par l'équation (2.11).

- En l'absence de bruit le signal maximal est obtenu à $\omega_{\mathrm{r}}$ pour les valeurs les plus basses possibles de l'impédance $Z_{\mathrm{r}}$ à la résonance dont l'expression

(*) Préamplificateur CDM 23 : Facteur de bruit $1 \mathrm{~dB}$, Avance et développement électronique, 2 bis, rue LéonBlum ZI 91120 Palaiseau, France. est donnée en (2.12). Le paramètre supplémentaire $L_{\mathrm{A}}$ donne une grande flexibilité pour les valeurs accessibles de $k$, coefficient de couplage du SQUID au circuit oscillant, qui n'est plus subordonné à la relation habituelle $k^{2} Q \simeq 1$.

- En revanche, l'effet du bruit sur la déformation des caractéristiques va à l'encontre du point précédent puisque l'amplitude du signal réel se stabilise à environ $50 \%$ de sa valeur théorique maximale (à $Z_{\mathrm{r}}=0$ ) pour toutes les valeurs de $Z_{\mathrm{r}}$ inférieures à l'impédance caractéristique de la ligne.

- La dépendance du signal en racine carrée de la fréquence de pompe $f_{\mathrm{P}}$ est conservée dans la limite de validité du modèle $\left(f_{\mathrm{P}} \ll R_{\mathrm{N}} / L_{\mathrm{S}}\right)$ soit environ $500 \mathrm{MHz}$ pour les dispositifs usuels. Au-delà, la dynamique de la jonction doit être intégralement prise en compte comme l'ont clairement montré E. Ben Jacob et Y. Imry [18] ou plus récemment R. I. Keranen et J. Kurkijärvi [19].

\section{Conclusion.}

L'analyse harmonique de la sonde classique, haute impédance, du SQUID rf couplé au circuit oscillant, complétée par une analyse temporelle qui précise la structure des plateaux de tension rend bien compte du fonctionnement à faible couplage du SQUID et à coefficient de qualité $Q$ du circuit élevé, pour des fréquences de pompe supérieures à une fréquence proche de l'accord. Pour les conditions plus générales de couplage du SQUID et d'attaque en fréquence, l'analyse temporelle présentée traite parfaitement la sonde classique en régime idéal non bruyant pourvu que la fréquence de pompe soit inférieure à la constante de temps $R_{\mathrm{N}} / L_{\mathrm{S}}$ du SQUID. Pour la sonde adaptée à basse impédance, la bobine d'adaptation augmente la complexité du circuit si bien que l'analyse harmonique est seule envisageable. L'analyse du bruit dans le système adapté a permis de différencier l'action du bruit intrinsèque du SQUID des autres sources : il est responsable d'environ $90 \%$ de la déformation des caractéristiques idéales ce qui permet de mesurer la probabilité de transition entre les états de flux total du SQUID. Ce résultat est cependant limité au SQUID largement hystérétique. Nous avons pu en déduire aisément la densité spectrale des fluctuations d'origine intrinsèque ; celle-ci valant $1,1 \times 10^{-5}$ $\phi_{0} / \sqrt{\mathrm{Hz}}$ est à comparer à la densité spectrale des fluctuations de bruit équivalent en flux ramené par le reste de la chaîne de mesure : $10^{-4} \phi_{0} / \sqrt{\mathrm{Hz}}$. En définitive, en dehors des avantages associés à l'électronique de détection et d'injection énoncés lors de l'introduction, la solution de la sonde adaptée procure une grande souplesse pour le choix du coefficient de couplage du SQUID au circuit oscillant mais n'apporte pas d'améliorations sensibles de la résolution en flux du capteur. 


\section{Bibliographie}

[1] Pascal, D. et Sauzade, M., J. Appl. Phys. 45 (1972) 474.

[2] DURET, D., Etude et réalisation d'un magnétomètre à effet Josephson exemples d'applications (thèse de docteur ingénieur université de Paris-Sud) 1976.

[3] LounAsmaA, O. V., Experimental principles and methods below $1 \mathrm{~K}$ (Academic press London and New-York) 1974, p. 140.

[4] Clarke, J., Supraconductors applications SQUIDs and machines (Brian B. Schwartz and Simon Foner) 1977, p. 90.

[5] Silver, A. H. and Zimmerman, J. E., Phys. Rev. B 57 (1967) 317-35.

[6] SCHWARTZ, M., Information transmission, modulation and noise (Mac Graw-Hill) 1980, p. 377-82.

[7] RoвbES, D., Optimisation du couplage d'un SQUID $R F$ à une ligne de réflectométrie : modèle théorique, influence $d u$ bruit de fond (thèse $3^{\mathrm{e}}$ cycle université de Caen) 1985, p. 44-7.

[8] Pascal, D., Contribution à l'étude de la détection de champs magnétiques par effet Josephson (Thèse d'état, université de Paris-Sud) 1981, p. 86-8.

[9] Likharev, K. K., Rev. Mod. Phys. 51 (1975) 101-59.

[10] Kurkijärvi, J., J. Appl. Phys. 44 (1973) 3729-33.

[11] Kurkijärvi, J. and WebB, W. W., Proc. Appl. superconductivity conf. Annapolis (I.E.E.E. New-York) 1972, p. 581-87.

[12] LikhareV, K. K., SQUID 80 (Berlin New-York, Walter de Gruyter and Co.) 1980, p. 474-503.

[13] Danilov, V. V. and LikHAREV, K. K., Radiotekh. Elektron. 25 (1980). Trad. : Radio Eng. Electron. Phys. 25, 8 (1980) 112-21.

[14] Belonogov, S. A., Snigirev, O. V., Tinchev, S. S. and LiKHAREV, K. K., Radiotekh. Elektron. 25 (1980). Trad. : Radio Eng. Electron. Phys. 25 (1980) 116-22.

[15] JaCkel, L. D. and Burhman, J. Low Temp. Phys. 19 (1975) 201.

[16] Robbes, D., Dubuc, C., Lepaisant, J., Bloyet, D. and DureT, D., I.E.E.E. Trans. Magn. 21 (1985) 892-95.

[17] Robbes, D., Dubuc, C., Lepaisant J., Bloyet, D. and DURET, D., SQUID'85 superconducting quantum interference devices and their application (Walter de Gruyter \& Co, Berlin New-York) 1985, p. 1071-76.

[18] Ben Jacob, E. and YMry, Y., J. Appl. Phys. 51 (1980) 4317-28.

[19] KerAnen, R. I. and KURKuÄrvi, J., J. Low Temp. Phys. 65 (1986) 279-88. 